International Journal of Biology, Pharmacy and Allied Sciences (IJBPAS) 'A Bridge Botuen caboratory and QRendo'

WWW.ijbpas.com

\title{
THE RELATION BETWEEN VITILIGO AND OTHER AUTOIMMUNE
}

\section{DISORDERS}

\begin{abstract}
ARFA $A^{1^{*}}$ AND ARFA $S^{2}$
1: Internal Medicine Trainee, West Hertfordshire Hospitals NHS Trust, UK

2: Royal Brompton Hospital, Cardiology Department, London

*Corresponding Author: E Mail: Ali.Arfa@doctors.net.uk; Phone: 00447438978550

Received 21 ${ }^{\text {st }}$ July 2019; Revised $6{ }^{\text {th }}$ Oct. 2019; Accepted $6{ }^{\text {th }}$ Jan. 2020; Available online $1^{\text {st }}$ June 2020

https://doi.org/10.31032/IJBPAS/2020/9.6.5063

ABSTRACT

Vitiligo is a common depigmenting skin disorder, characterized by acquired, idiopathic, progressive, circumscribed hypomelanosis of the skin and hair, with total absence of melanocytes microscopically. It occurs worldwide, with an incidence rate of between $0.1 \%$ and $2 \%$. Vitiligo is an important skin disease having a major impact on the quality of life of the patient suffering from it. The causes of this condition are uncertain but seem to be dependent on the interaction of genetic, immunological and neurological factors. Vitiligo coexists with other autoimmune disorders, Sutton or halo nevus, and malignant melanoma. The substantial disfigurement associated with vitiligo can cause serious emotional stress for the patient, which necessitates treatment. Vitiligo is associated with numerous autoimmune, systemic and dermatological diseases such as thyroid disease, alopecia areata, diabetes mellitus, pernicious anemia, systemic lupus erythematosus, rheumatoid arthritis, Addison's disease, inflammatory bowel disease, dermatomyositis, scleroderma, ocular and audiological abnormalities, psoriasis, and atopic dermatitis. It is essential to increase awareness of these comorbidities in order to improve the disease burden and quality of life of patients with vitiligo. In this review paper, we reviewed correlation of vitiligo with these diseases.
\end{abstract}

Keywords: Vitiligo, Comorbid autoimmune, systemic and dermatological diseases 


\section{INTRODUCTION}

Vitiligo affects all races and has a long history. It has been known for thousands of years because of its visual phenotype. It is a common depigmenting skin disorder, characterized by acquired, idiopathic, progressive, circumscribed hypomelanosis of the skin and hair, with total absence of melanocytes microscopically. Vitiligo is a psychologically devastating and frequently recalcitrant skin disorder [1]. The causes of this condition are uncertain but seem to be dependent on the interaction of genetic, immunological and neurological factors. In general, it shows multifactorial etiology and polygenic inheritance [2]. It is essential to increase awareness of these comorbidities in order to improve the disease burden and quality of life of patients with vitiligo. In this review papers, we reviewed correlation of vitiligo with these diseases.

\section{MATERIAL AND METHODS}

The keywords used for the literature search for this review was peer-reviewed articles following keywords:"Vitiligo", "Comorbid autoimmune", "systemic and dermatological diseases", "thyroid disease", "alopecia areata", "diabetes mellitus", "pernicious anemia", "systemic lupus erythematosus", "rheumatoid arthritis", "Addison's disease", "inflammatory bowel disease", "dermatomyositis", "scleroderma", "audiological abnormalities", "psoriasis", and atopic dermatitis". The search was done and almost 88 abstract and papers collected which the keywords included in them. Among them, the papers were fit the criteria selected and available full-text articles read. Related articles were also scrutinized. Hand search was also driven. The search carried out using Biological Abstracts, Chemical Abstracts, and the data bank of the PubMed and Medline database updated to 2020. The references found in the search were then studied in detail.

\section{Epidemiology}

Vitiligo is the most prevalent pigmentary disorder, occurs worldwide, with an incidence rate of between $0.1 \%$ and $2 \%$, irrespective of age, race, ethnic origin or skin color. The incidence of vitiligo in those with racially pigmented skin is higher, although reliable figures are not available. The prevalence has been reported as high as $4 \%$ in some South Asian, Mexican and US populations. Both sexes are equally afflicted. In some studies, a female preponderance has been reported but the discrepancy has been attributed to a presumed increase in reporting of cosmetic concerns by female patients [3]. Although familial clustering of cases is commonly seen, inheritance occurs in a nonMendelian pattern. Occasionally, it is reported that vitiligo is determined by an autosomal dominant gene of variable 
penetrance. It has also been reported in monozygotic twins. Vitiligo commonly begins in childhood or young adulthood with peak onset of 10-30 years, but it can develop at any age. Several studies report that $50 \%$ of cases appear before the age of 20 years. It is rarely seen in infancy or old age. The incidence decreases with increasing age [4].

Vitiligo is a multifactorial disorder, related to both genetic and non-genetic factors. It is generally agreed that there is an absence of functional melanocytes in vitiligo skin and that this loss of histochemically recognizable melanocytes is the result of destruction. Due to the observed variation in clinical manifestations of the disease, it seems likely that etiology of vitiligomay differs among patients and is complex [5]. Therefore, several theories on vitiligo etiopathogenesis have been combined to formulate a convergence theory of vitiligo. Segmental vitiligo frequently occurs in a dermatomal pattern, leading to a neural hypothesis. This hypothesis proposes that elevated levels of some neurotransmitters and catecholamine degrading enzymes injure melanocytes [6]. Vitiligo patients tend to have higher scores for anxiety, depression, adjustment disorders, obsessive symptoms and hypochondria. Thus, there may be a relationship between stress and the development of vitiligo. [7] indicated that psychological stress increases levels of neuroendocrine hormones, affects the immune system and alters the level of neuropeptides, which may be the initial steps in the pathogenesis of vitiligo [7]. Many patients with generalized vitiligo have serum autoantibodies and circulating autoreactive $\mathrm{T}$ cells directed against melanocytes and melanocyte components, and a careful analysis of margins of active generalized vitiligo lesions have repeatedly shown sparse infiltrates of cytotoxic $T$ cells. [8] found an elevated ratio of CD4+ /CD8 $+\mathrm{T}$ cells to be a sign of imbalanced lymphocyte immune response in vitiligo patients, but they did not find evidence for a pathological distribution of $B$ cells in peripheral blood within their patients. In addition, increased levels of soluble interleukin (IL)-2 receptor, IL-6 and IL-8 has been found in vitiligo patients, which further suggests that T-cell activation may be a component in vitiligo pathogenesis. The detection of significantly higher expression of IL-6 and tumor necrosis factor (TNF)-ain vitiligo skin, compared with healthy skin indicates an imbalance of epidermal cytokines at sites of lesions [9].

\section{Vitiligo and other systemic disorders}

Association between vitiligo and thyroid disorders Thyroid functional disorders and autoimmune thyroid diseases have been reported in association with vitiligo and it seems that the incidence of clinical and 
subclinical thyroid involvement is more common in vitiligo patients than healthy subjects [3]. Vitiligo might be connected with an increased activity of the thyroid gland. A distinct rise of the basal metabolism in half of patients tested [10]. In addition, there is also a study reporting a significantly increased prevalence of vitiligo in patients with autoimmune thyroid disease compared with patients with non-autoimmune thyroid disease. The association of autoimmune thyroid disease with HLA genes has received much attention and in several populations of different ethnic background an association between the HLA system and Graves' disease was reported [10]. Several genes outside the HLA system have been found to be associated with autoimmunethyroiditis. Hashimoto thyroiditis and Graves' disease are the most important and prevalent autoimmune thyroid diseases associated with vitiligo [11]. Various thyroid autoantibodies, including thyroid stimulating antibody, anti-thyroglobulin antibody and anti-thyroid peroxidase antibody, are detectable in autoimmune thyroid diseases, the latter being themost sensitive test for the diagnosis and follow up of this group of this group of diseases [12]. An association of vitiligo with other autoimmune diseases was found in $6 \%$ of patients. Moreover, increased risk of autoimmune/endocrine diseases was shown in first- and second-degree relatives of vitiligo patients with positive organspecific antibodies. Almost all of the patients with vitiligo and other autoimmune diseases had generalized vitiligo. Among them, 31\% had autoimmunethyroid disease, $14 \%$ had rheumatoid arthritis and $12 \%$ had type 1 diabetes mellitus [12]. [10] showed no statistically significant association between vitiligo and Addison's disease ordiabetes mellitus type 1 . They showed that these diseases are much rarer than autoimmune thyroiditis and the number of their cases with these two diseases are far above the reported frequencies in healthy populations [10].

\section{Alopecia areata}

Alopecia areata is an autoimmune disease that is characterized by patchy or total hair loss of the scalp hair and sometimes universal loss of all body hair. 46 A sizable proportion of vitiligo patients have simultaneous alopecia areata. In a study involving 133 patients with generalized vitiligo, alopecia areata was documented in $5.3 \%$, which, according to the authors, is in accordance with results from Africa, China, and India [13]. Another study found alopecia areata to be the disease most significantly associated with vitiligo, while [14] found it to be the second most common autoimmune disease in their study 
group, highlighting a possible shared pathogenesis of the two diseases. Notably, a very high frequency of alopecia areata of $12.5 \%$ was observed in a study involving 80 vitiligo patients in Turkey. Other studies also report a significant association between vitiligo and alopecia areata with overall rates ranging from 0.5 to $12.5 \%$ [15].

\section{Diabetes mellitus}

Many studies have identified a possible correlation between vitiligo and diabetes mellitus. Approximately $1-7 \%$ of vitiligo patients have diabetes mellitus. 45 This prevalence is significantly higher than the overall population prevalence of diabetes of 0.674\%. Type 1 diabetes (insulindependent) is an autoimmune disease, and the coexistence of the two diseases lends support to the autoimmune theory of vitiligo and the possibility that vitiligo, too, is a T-cell-mediated disease. Furthermore, higher levels of inflammatory cytokines in patients with vitiligo and concomitant type 1 diabetes compared to patients with only vitiligo and controls suggest a possible inflammatory link between the two diseases [16]. In a study of 6,516 vitiligo probands, [16] found significantly increased prevalence of diabetes mellitus in familial vitiligo probands $(3.3 \%)$ but not in the total population of probands. Such data is strongly in favor of a genetically determined susceptibility to vitiligo and specific autoimmune diseases in familial vitiligo compared to sporadic vitiligo.

\section{Inflammatory bowel disease}

Increased frequency of IBD in vitiligo patients has been reported in some studies. Both [14] have observed significant associations between IBD and vitiligo with prevalence rates of 0.9 and $2.3 \%$, respectively. These results are consistent with previous findings by [17] in a survey of Caucasian vitiligo patients and their first-degree relatives in which an increased prevalence of IBD was observed among the probands but not the relatives. Of the probands, $0.67 \%$ were affected compared with a population prevalence of $0.37 \%$.

\section{Pernicious anemia}

Pernicious anemia is frequently reported in vitiligo patients. [18] observed a significant increase in the frequency of pernicious anemia in their study group of 300 patients with vitiligo; the prevalence of pernicious anemia was $1.3 \%$, which is significantly above the estimated population prevalence of $0.15 \%$. Again, Turkey has reported increased rates of pernicious anemia in their study group with a prevalence of $8.7 \%, 28$ whereas [14] found a rate of $0.5 \%$. In contrast, no significant increase in the frequency of pernicious anemia in patients with vitiligo was observed by other studies including [19] who found no association despite specific search for the condition. 


\section{Systemic lupus erythematosus}

[14] found the prevalence of SLE in a cohort of 1,098 vitiligo patients to be $0.3 \%$. When stratified by race (black, white, other), SLE was only observed among black patients. Moreover, [20] found a significant association between vitiligo and SLE, which also was more pronounced in women and elderly patients.

\section{Rheumatoid arthritis}

Some studies find a higher frequency of RA among vitiligo patients. [19] reported that $2.9 \%$ of their vitiligo patients had RA and that it was most common in AfricanAmerican/Black patients. This is in agreement with data reported by [16] in which the frequency of RA was significantly increased in both generalized vitiligo probands and their first-degree relatives, 2.2 and $0.59 \%$, respectively, compared to the general population prevalence of $0.34 \%$. The highest prevalence of $3.8 \%$ was reported by [21] in a study of 133 probands with familial vitiligo.

\section{Vitiligo and vitamin D metabolism}

Accumulating data give rise to a link with vitamin $\mathrm{D}$ in the etiopathogenesis of vitiligo. Vitamin D exerts its effects through the nuclear vitamin $\mathrm{D}$ receptor (VDR). There are studies showing an association between VDR gene polymorphisms and vitiligo that avoids the increased risk for developing vitiligo [22].
[23] detected highly significant 25(OH)D deficiency in patients with vitiligo when compared to healthy controls. However, they did not observe any significant correlation between associated autoimmune diseases and the serum $25(\mathrm{OH}) \mathrm{D}$ levels of patients. [24] investigated the serum $25(\mathrm{OH}) \mathrm{D}$ deficiency in Chinese patients with vitiligo. Their data did not reveal a correlation between vitamin $\mathrm{D}$ levels and onset of vitiligo. On the other hand, they demonstrated that patients with vitamin D deficiency were at an increased risk of developing autoimmune diseases. The exact cause of melanocyte loss in vitiligo is still debatable. It is considered to be the result of an autoimmune-mediated process, including both humoral (circulating antibodies targeting melanocyte antigens) and cellular immune aberrations leading to the destruction of melanocytes. Vitiligo is associated with overproduction of proinflammatory cytokines such as TNF$\alpha$, IL-6, and IL-2 [25]. A less traditional function of vitamin $D$ has been demonstrated as immunoregulation, including the regulation and differentiation of the cells of both the innate and adaptive immune systems [26].

The female preponderance in our study 1.6:1 is in accordance with certain other studies in this regard which confirm female preponderance. The higher frequency of 
Vitiligo in females is in consistence with certain other studies in this regard [27]. The association between vitiligo and autoimmune thyroiditis (Hashimoto's disease) is well documented in adults and children. The association found with thyroid disorders was less in our study as compared to another study in which the association with autoimmune thyroiditis in adults had been around $30 \%$, amongst the vitiligo patients [28]. The other autoimmune disorders associated with vitiligo in our study were alopecia areata, diabetes mellitus, and Rheumatoid arthritis. These findings are similar to certain similar studies that analyzed the association between vitiligo and other autoimmune diseases [29]. These associations are indicative of the fact that vitiligo shares a common genetic etiologic links with these other autoimmune disorders. The immune system is undeniably involved in the pathogenesis, as evidenced by the effectiveness of immuno-modulatory agents, such as corticosteroids and calcineurin inhibitors in the treatment of vitiligo [29].

\section{CONCLUSION}

There is strong association of vitiligo with other autoimmune disorders and has a positive family history indicating that both genetic as well as non-genetic autoimmune component in the etiology of vitiligo. Patients having vitiligo should be looked for other autoimmune disorders especially the thyroid disorders.

\section{REFERENCES}

[1] Birlea SA, Fain PR, Spritz RA. A Romanian population isolate with high frequency of vitiligo and associated autoimmune diseases. Arch Dermatol., 2008; 144: 310316.

[2] Jin Y, Riccardi SL, Gowan K, Fain PR, Spritz RA. Finemapping of vitiligo susceptibility loci on chromosomes 7 and 9 and interactions with NLPR1 (NALP1). J Invest Dermatol., 2010; 130: 774 783.

[3] Daneshpazhooh M, Mostofizadeh GM, Behjati J, Akhyani M, Mahmoud Robati R. Anti-thyroid peroxidase antibody and vitiligo: a controlled study. BMC Dermatol., 2006; 6: 3.

[4] Szczurko O, Boon HS. A systematic review of natural heath product treatment for vitiligo. BMC Dermatol., 2008; 8: 2.

[5] Yaghoobi R, Omidian M, Bagherani N. Vitiligo: A review of the published work. Journal of Dermatol., 2011; 38: 419-431.

[6] Namazi MR. Phnytoin as a novel anti-vitiligo weapon. J Autoimmune Dis., 2005; 2: 11. 
[7] Aghaei SH, Sodaifi M, Jafari P, Mazharinia N, Finlay AY. DLQI scores in vitiligo: reliability and validity of the Persian version. BMC Dermatol., 2004; 4: 8.

[8] Pichler R, Sfetsos K, Badics B, Gutenbrunner S, Berg J, Aubo“ ck J. Lymphocyte imbalance in vitiligo patients indicated by elevated CD4+/CD8+ T-cell ratio. Wien Med Wochenschr., 2009; 159: 337-341.

[9] Le Poole IC, Luiten RM. Autoimmune etiology of generalized vitiligo. Curr Dir Autoimmun., 2008; 10: 227-243.

[10] Zettinnig G, Tanew A, Fischer G, Mayr W, Dudczak R, Weissel M. Autoimmune disease in vitiligo: do antinuclear antibodies decrease thyroid volume? Clin. Exp. Immunol., 2003; 131: 347-354.

[11] Tomer Y, Barbesino G, Greenberg DA, Concepcion E, Davies TF. Mapping the major susceptibility loci for familial Graves' and Hashimoto's diseases: evidence for genetic heterogeneity and gene interactions. J Clin. Endocrinol. Metab., 1999; 84: 4656-4664.

[12] Ai J, Leonhardt MJ, Heymann RW. Autoimmune thyroid disease. Etiology, pathogenesis, and dermatologic manifestations. J Am
Acad. Dermatol., 2003; 48: 641659.

[13] Narita T, Oiso N, Fukai K, et al. Generalized vitiligo and associated autoimmune diseases in Japanese patients and their families. Allergol. Int., 2011; 60: 505-508.

[14] Gill L, Zarbo A, Isedeh P, et al. Comorbid autoimmune diseases in patients with vitiligo: a crosssectional study. J Am Acad. Dermatol., 2016; 74: 295-302.

[15] Poojary SA. Vitiligo and associated autoimmune disorders: a retrospective hospital-based study in Mumbai, India. Allergol. Immunopathol (Madr)., 2011; 39: 356-361.

[16] Zhang Z, Xu SX, Zhang FY, et al. The analysis of genetics and associated autoimmune diseases in Chinese vitiligo patients. Arch Dermatol Res., 2009; 301: 167173.

[17] Alkhateeb A, Fain PR, Thody A, et al. Epidemiology of vitiligo mand associated autoimmune diseases in Caucasian probands and their families. Pigment Cell Res., 2003; 16: 208-214.

[18] Sawicki J, Siddha S, Rosen C. Vitiligo and associated autoimmune disease: retrospective 
review of 300 patients. J Cutan

Med Surg., 2012; 16: 261-266.

[19] Sheth VM, Guo Y, Qureshi AA. Comorbidities associated with vitiligo: a ten-year retrospective study. Dermatol., 2013; 227: 311315.

[20] Chen YT, Chen YJ, Hwang CY, et al. Comorbidity profiles in association with vitiligo: a nationwide population-based study in Taiwan. J Eur. Acad. Dermatol. Venereol., 2015; 29: 1362-1369.

[21] Laberge G, Mailloux CM, Gowan $\mathrm{K}$, et al. Early disease onset and increased risk of other autoimmune diseases in familial generalized vitiligo. Pigment Cell. Res., 2005; 18: 300-305.

[22] Li K, Shi Q, Yang L, Li X, Liu L, Wang L, Li Q, Wang G, Li CY, Gao TW. The association of vitamin D receptor gene polymorphisms and serum 25hydroxyvitamin D levels with generalized vitiligo. $\mathrm{Br} \quad \mathrm{J}$ Dermatol., 2012; 167: 815-821.

[23] Banerjee K, Barbhuiya JN, Ghosh AP, Dey SK, Karmakar PR. The efficacy of low-dose oral corticosteroids in the treatment of vitiligo patient. Indian J Dermatol. Venereol. Leprol., 2003; 69: 135137.
[24] Xu X, Fu WW, Wu WY. Serum 25-hydroxyvitamin D deficiency in Chinese patients with vitiligo: a case-control study. PLoS One., 2012; 7: e52778.

[25] Ongenae K, Van Geel N, Naeyaert JM. Evidence for an autoimmune pathogenesis of vitiligo. Pigment Cell Res., 2003; 16: 90-100.

[26] Arnson Y, Amital H, Shoenfeld Y. Vitamin D and autoimmunity: new aetiological and therapeutic considerations. Ann. Rheum. Dis., 2007; 66: 1137-1142.

[27] Zum B, Alam M, Khondker L, Siddiqua A, Alam MN, et al. (2013) Association of other Autoimmune Diseases in Vitiligo Patients. Community Based Medical Journal 02: 57-61.

[28] Yang $Y$, Lin $X, F u$ W, Luo X, Kang K (2010) An approach to the correlation between vitiligo and autoimmune thyroiditis in Chinese children. Clin. Exp. Dermatol., 35: 706-710.

[29] Lotti T, Gori A, Zanieri F, Colucci R, Moretti S (2008) Vitiligo: new and emerging treatments. Dermatol. Ther., 21: 110-117. 